\title{
Visual contrast sensitivity in tonic-clonic epileptic patients
}

\author{
Paloma C. Bezerra ${ }^{1}$, Caroline C. G. Alencar ${ }^{2}$, Sandra R. Mousinho ${ }^{1}$ and Natanael A. dos Santos ${ }^{1}$ \\ 1 - Universidade Federal da Paraíba, João Pessoa, PB, Brazil \\ 2 - Universidade de São Paulo, São Paulo, SP, Brazil
}

\begin{abstract}
We measured the effects of epilepsy on visual contrast sensitivity to linear and vertical sine-wave gratings. Sixteen female adults, aged 21 to 50 years, comprised the sample in this study, including eight adults with generalized tonic-clonic seizure-type epilepsy and eight age-matched controls without epilepsy. Contrast threshold was measured using a temporal two-alternative forced-choice binocular psychophysical method at a distance of $150 \mathrm{~cm}$ from the stimuli, with a mean luminance of $40.1 \mathrm{~cd} / \mathrm{m}^{2}$. A one-way analysis of variance (ANOVA) applied to the linear contrast threshold showed significant differences between groups $(F[3,188]=14.829 ; p<.05)$. Adults with epilepsy had higher contrast thresholds $(1.45,1.04$, and 1.18 times for frequencies of $0.25,2.0$, and 8.0 cycles per degree of visual angle, respectively). The Tukey Honestly Significant Difference post hoc test showed significant differences $(p<.05)$ for all of the tested spatial frequencies. The largest difference between groups was in the lowest spatial frequency. Therefore, epilepsy may cause more damage to the neural pathways that process low spatial frequencies. However, epilepsy probably alters both the magnocellular visual pathway, which processes low spatial frequencies, and the parvocellular visual pathway, which processes high spatial frequencies. The experimental group had lower visual contrast sensitivity to all tested spatial frequencies. Keywords: epilepsy, tonic-clonic seizures, contrast sensitivity,visual pathways.
\end{abstract}

Received 23 February 2011; received in revised form 16 May 2011; accepted 30 May 2011. Available on line 15 June 2011

\section{Introduction}

Epilepsy is a chronic neurological disorder, but no consensus has been reached about its accurate definition (Guerreiro \& Guerreiro, 1996). It is characterized by recurrent and uncontrollable seizures that occur as a result of excessive electrical discharges in a group of brain cells. This sudden and usually brief alteration in brain activity is reversible, and different parts of the brain can elicit such discharges (Fonseca \& Tedrus, 2004; Kaplan \& Sandock, 1997; Lorenzato, Cavalli, \& Duarte, 2002).

According to the World Health Organization (WHO, 2010), 50 million people in the world have epilepsy, with 40 million living in developing countries. Epilepsy can affect every age group, but it especially affects children younger than 2 years of age

Paloma C. Bezerra, Sandra R. Mousinho, and Natanael A. dos Santos, Laboratório de Percepção, Neurociências e Comportamento (LPNeC), Universidade Federal da Paraíba, João Pessoa, PB, Brazil. Caroline C. G. Alencar, Programa de Neurociências e Comportamento, Instituto de Psicologia, Universidade de São Paulo (USP), São Paulo, SP, Brazil. Correspondence regarding this article should be directed to: Caroline Costa Gomes Alencar, Departamento de Fisiologia e Biofísica Instituto de Ciências Biomédicas-ICB Universidade de São Paulo (USP), Av. Prof. Lineu Prestes, 1524, Room 239, São Paulo, SP 05508-900 Brazil. Phone: +55 1130917242. Fax: 5511 30917426. E-mail: carolalencar@usp.br and the elderly above 65 years of age (Santo, Maineri, \& Portuguez, 2004).

Epilepsy can be classified into two main types: partial seizures and general seizures (Pinel, 2005). Partial seizures begin with an electrical discharge in one limited area of the brain, whereas general seizures begin with widespread electrical discharge that involves both sides of the brain at once. Epilepsy with general seizures can be divided into absence seizures, myoclonic seizures, tonic seizures, clonic seizures, and tonic-clonic seizures (Tedrus $\&$ Fonseca, 2005). Epilepsy with tonic-clonic seizures is the subtype with the highest prevalence (WHO, 2010). During tonic-clonic seizures, the patient initially presents a loss of conscience and falls, exhibiting rigidity shortly thereafter, and the extremities of the body begin to shake and contract (Guerreiro \& Guerreiro, 1996).

Patients with epilepsy usually have visual problems that can be attributable to the pathology itself or to the drugs used to control seizures (Hilton, Cubbidge, Hosking, Betts, \& Comaish, 2002; Mirabella et al., 2007; Verrotti, Manco, Matricardi, Franzoni, \& Chiarelli, 2007). However, many studies have related epilepsy to visual perception, but these studies used different methodologies and samples that were poorly described only as patients with epilepsy or partial epilepsy (Hilton et al., 2002; Sorri, Rissanen, Mantyjarvi, \& Kalviainen, 2005; Sorri, Kalviainen, \& Mantyjarvi, 2005). 
For example, Mirabela et al. (2007) developed a study that investigated visual deficits in children, with seizuretype epileptic infants either taking no medicine, receiving vigabatrin (VGB), or taking other antiepileptic drugs. They measured contrast sensitivity (CS) using the sweep visual evoked potential method. They found that the decreased CS was not related to VGB but rather to epileptic seizures because the deficit began before the administration of the drug and did not become worse with VGB.

In two studies, Sorri et al. tested CS in patients with partial epilepsy treated with tiagabine monotherapy (Sorri et al., 2005a) and valproate monotherapy (Sorri et al., 2005b). Both studies found no changes in CS.

Tomson, Nilsson, and Levi (1988) measured CS in epileptic patients treated with carbamazepine to verify whether this drug could reduce psychophysical CS. They found a reduction in CS in the epileptic group treated with carbamazepine compared with the control group (i.e., without epilepsy). No conclusion could be made from these results, however, that the decrease in CS was attributable to carbamazepine or epilepsy. This difficulty separating the effects of medications from the effects of epilepsy is very common in the literature (Verrotti et al., 2007).

The aim of the present study was to examine the effects of tonic-clonic seizure-type epilepsy on visual CS using the psychophysical staircase method with forced choice between two temporal alternatives. Previous studies investigated other epilepsy types and used psychophysical methods that did not minimize the effects of the criteria of the participants' response choice (GarcíaPérez, 1998; Schwartz, 2004). Therefore, this may be one reason why the psychophysical forced-choice method is one of the most widely used methods in the literature.

\section{Methods}

\section{Participants}

Sixteen female volunteers, aged 21 to 50 years (mean $=26.6 ; S D=7.5$ ), comprised the sample, including eight adults with congenital generalized tonic-clonic seizure-type epilepsy (experimental group [EG]) and eight age-matched controls without epilepsy (control group [CG]). The volunteers from the EG were taking carbamazepine, phenobarbital, and oxicarbazepine (for more information, see Yacubian [1999] and Garzon [2002]) for at least 4 years. All of the volunteers had normal visual acuity $(6 / 6$, inclusion criterion) tested with directional "E" Rasquin optotype cards (Xenonio, São Paulo, SP, Brazil) and were free of eye or neurological diseases (exclusion criterion), with the exception of epilepsy in the EG. The participation of all volunteers adhered to ethical considerations relevant to research that involves human subjects according to resolution 196/96 of the National Health Council, Health Ministry, Brazil. The experimental design was approved by the Ethics Committee of the Health
Sciences Centre, Universidade Federal da Paraíba. The subjects' participation was voluntary and commenced after they signed an informed consent.

Adults with tonic-clonic epileptic seizures were diagnosed by neurologists and electroencephalographic tests. These patients were selected at the Hospital Universitário Lauro Wanderley, a public hospital at the Universidade Federal da Paraíba, Brazil.

\section{Visual stimuli}

The achromatic and circular stimuli had a diameter of approximately $7.2^{\circ}$ of the visual angle and were generated in grayscale, with an average luminance of $40.1 \mathrm{~cd} / \mathrm{m}^{2}$. The neutral stimulus was gray and contained only the average luminance. The test stimuli were linear, vertical, and static sine-wave gratings (Campbell \& Green, 1965) and were defined in Cartesian coordinates $(0.25,2.0$, and 8.0 cycles per degree of visual angle [cpd]) as the following:

$$
\mathrm{L}(\mathrm{x})=\mathrm{Lm}[1+\operatorname{csin}(2 \pi \mathrm{fx}+\phi)] \mathrm{Eq} .1
$$

$\mathrm{L}(\mathrm{x})$ is the luminance at a point on the sine-wave. $\mathrm{Lm}$ is the average luminance. $\mathrm{c}$ is the contrast. $\mathrm{f}$ is frequency in cpd. $\Phi$ is the spatial phase. In this case, contrast is defined by the following formula (Michelson, 1891):

$$
\mathrm{C}=\frac{\mathrm{Lmax}-\mathrm{Lmin}}{\mathrm{Lmax}+\mathrm{Lmin}} \mathrm{Eq} .2
$$

$\mathrm{C}$ is the contrast. Lmax is the maximum luminance. Lmin is the minimum luminance.

\section{Equipment}

The stimuli were set to appear in the center of the video monitor, $150 \mathrm{~cm}$ from the observer (Figure 1). A fixed chair and forehead-chin support were used to control the distance. The monitor used to generate and present the stimuli was a color cathode ray tube (CRT) with a 19-inch screen, $1024 \times 768$ resolution, and $70 \mathrm{~Hz}$ image update frequency controlled by a microcomputer through VGA and DVI video connectors.

The dynamic range proportion of the monitor was expanded more than 64 times by a BITS ++ digital video processor (Cambridge Research Systems, Rochester, UK). The 8 bits per channel or 24 bits per pixel that exist in common monitors, equivalent to 256 levels in grayscale, were expanded to 14 bits per channel or 42 bits per pixel, equivalent to more than 16,384 levels in grayscale, enabling the use of a computer to run the experiments with high-contrast resolution. BITS++ worked with software developed by our group, written in $\mathrm{C}++$ language, to generate the stimuli, control the presentation, and record the contrast threshold values.

The LightScan program with an Optical photometer (Cambridge Research Systems) measured screen 
luminance and performed gamma correction using all 256 points from $0-255$ as the sample (gamma $=1.8$ ), which is indicated when using BITS++. The minimum and maximum luminance values of the screen were $0.20 \mathrm{~cd} / \mathrm{m}^{2}$ and $80.0 \mathrm{~cd} / \mathrm{m}^{2}$, respectively (average luminance, $\left.40.1 \mathrm{~cd} / \mathrm{m}^{2}\right)$. The background luminance was the minimum luminance itself. The laboratory room measured $2.5 \times 2.0 \mathrm{~m}$ and was illuminated by one Philips $20 \mathrm{~W}$ fluorescent lamp. The walls were gray to enable better control of ambient lighting during the experiment.

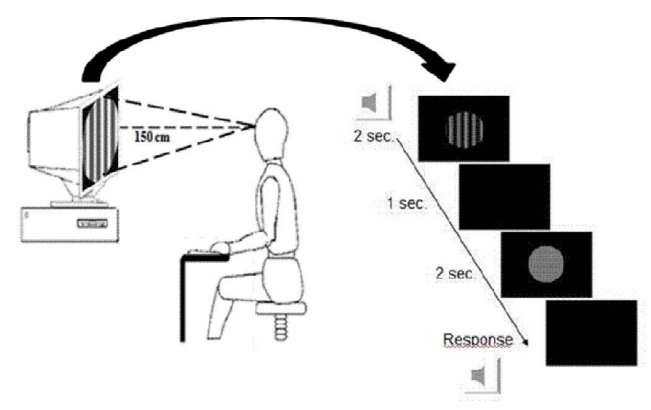

Figure 1. Stimulus presentation scheme. The stimuli were originally calibrated to be seen at a distance of $150 \mathrm{~cm}$ on a 19-inch display.

\section{Procedure}

The visual CS of all participants was estimated using the psychophysical staircase method, with a forced choice between two temporal alternatives as originally proposed by Wetherill and Levitt (1965). The forced-choice method is based on the probability of consecutive hits by the volunteer (i.e., approximately 100 opportunities to choose between two stimuli). In this case, with each pair of stimuli, one only had the mean luminance ( $40.1 \mathrm{~cd} / \mathrm{m}^{2}$; i.e., neutral stimulus), and the other contained one of the spatial frequencies (i.e., test stimulus). The test stimuli (sinusoidal vertical linear sine-wave gratings) are seen approximately $79 \%$ of the time by the volunteer.

The procedure for measuring the threshold for each frequency consisted of presenting successive pairs of simple stimuli, in which one was the test stimulus that should be identified by the participant (the first or second stimulus of each pair). The order of the presentation of the stimuli and frequencies was random and controlled by the software. The criterion used to measure the CS for each spatial frequency was three consecutive hits to decrease $20 \%$ of the remaining contrast and an error of increasing contrast by the same percentage.

In this type of procedure, the number of presentations of the stimulus pairs is variable and depends on the success of the volunteer and the number of previously defined threshold values. In the present study, each experimental session ended automatically after six threshold values (three maximum and three minimum values due to the staircase method) or six reversals obtained by the volunteer, which took an average of 5 to $10 \mathrm{~min}$.

A stimulus sequence was presented during each experimental session, beginning with a beep followed immediately by the presentation of the first stimulus for $2 \mathrm{~s}$. A $1 \mathrm{~s}$ interval occurred between stimuli, followed by the presentation of the second stimulus for $2 \mathrm{~s}$ and the volunteer's response. A correct response by the volunteer was followed by another beep. The interval between trials was $3 \mathrm{~s}$, regardless of whether the answer (or choice) was correct or wrong. The beeps that indicated the beginning of the stimulus pair presentation and the correct choice were different.

All participants received the following statement: "Pairs of circles will appear on the screen, one after the other. One of them will be totally gray, whereas the other will contain light and dark stripes. When the circle with stripes appears first, you must press the left mouse button (button 1); when the circle with stripes appears in the second place, you should press the right mouse button (button 2)."

Notably, each session began with the test stimulus with contrast at the supra-threshold level, and the experiments only began when the experimenter was sure that the participants understood and responded according to the directions. In this context, instructions were repeated in a training and familiarization session under the experimental conditions. All measurements were performed with binocular vision and normal pupils.

After each experimental session, the software produced a record sheet with the threshold contrast values, six in the test and six in the retest. Values were grouped according to the experimental condition (i.e., adults with epilepsy [EG] and adults without epilepsy [CG]).

\section{Results}

To perform a parametric analysis, we confirmed that the threshold values for both groups had a normal distribution using Kolmogorov-Smirnov's test and homogeneity of variance using Levine's test. The outlier values were considered missing case using Tukey's outlier test. The median contrast threshold values for each participant was then used for the data analyses.

One-way analysis of variance (ANOVA) applied to visual CS showed significant differences between groups $(F[3,188]=14.829 ; p<.05)$. Adults with epilepsy had higher contrast thresholds that reached approximately $1.45,1.04$, and 1.18 times for the frequencies of $0.25,2.0$, and $8.0 \mathrm{cpd}$, respectively (Figure 2). The Tukey Honestly Significant Difference post hoc test showed significant differences $(p<.05)$ for all of the tested spatial frequencies. 


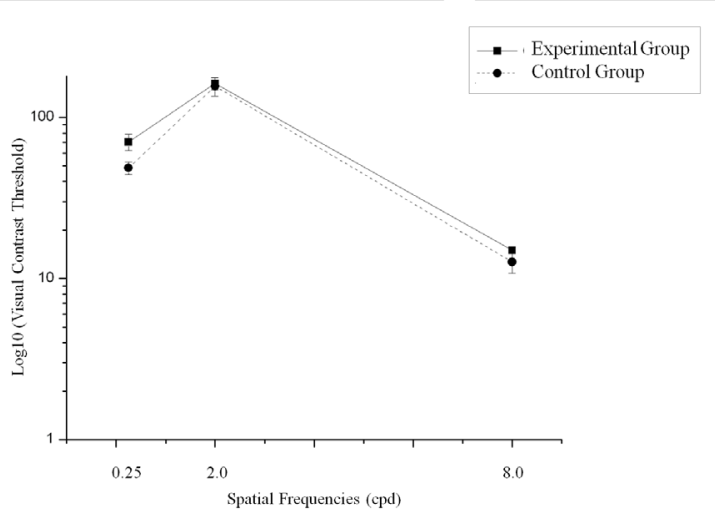

Figure 2. The estimated visual contrast threshold in female adult volunteers is shown as a spatial frequency function of $0.25,2.0$, and $8.0 \mathrm{cpd}$. The mean error bars were corrected to the sample's size to represent a $95 \%$ confidence interval. The continuous curve (一- - ) represents the contrast threshold of adults with generalized tonic-clonic seizure-type epilepsy (experimental group), and the dotted curve (------) represents the contrast threshold of adults without epilepsy (control group).

\section{Discussion}

The present study measured luminance CS in participants without any neuropsychiatric disease and patients with epilepsy using the psychophysical staircase method with forced choice between two options at a photopic luminance level. The aim of this research was to verify whether epilepsy alters the response of the visual system or basic sensory mechanisms related to the processing of luminance contrast in sine-wave gratings of $0.25,2.0$, and $8.0 \mathrm{cpd}$.

The present study was based on the hypothesis that most diseases that change or affect the nervous system, including epilepsy, cause structural, functional, and behavioral changes in humans (Hilton et al., 2002). Consistent with this hypothesis, visual neuroscience research uses visual CS to evaluate and diagnose changes in optic and neural structures related to diseases that are not directly related to visual changes, such as depression (Wesner \& Tan, 2006), schizophrenia (Slaghuis \& Thompson, 2003), and malnutrition (Santos, Alencar, \& Dias, 2009; Santos \& Alencar, 2010), among others.

The results showed that visual CS in the CG and EG had similar patterns, with maximum sensitivity to the medium spatial frequency $(2.0 \mathrm{cpd})$ and a decrease in visual CS in response to the lowest spatial frequency $(0.25)$ and highest spatial frequency $(8.0 \mathrm{cpd})$. However, the statistical analyses revealed significant differences between groups at all of the tested spatial frequencies $(0.25,2.0$, and $8.0 \mathrm{cpd})$. The largest difference between groups was with the lowest spatial frequency. Patients with epilepsy needed 1.5 more contrast to detect it. Therefore, epilepsy may cause more damage to the neural pathways that preferentially process low spatial frequencies. According to Souza, Gomes, Saito, da Silva
Filho, and Silveira (2007), at low spatial frequencies (i.e., $0.4-0.8 \mathrm{cpd}$ ), only the magnocellular visual pathway appears to be relevant for the cortical response. However, because the EG had lower visual CS in response to all tested spatial frequencies, epilepsy may alter both the magnocellular visual pathway, which preferentially processes low spatial frequencies, and parvocellular visual pathway, which preferentially processes high spatial frequencies (Burbeck \& Kelly, 1981). Lee (2011) stated that the way in which central pathways process afferent inputs in more complex spatial contexts makes such correlations less clear, but the identification of psychophysical luminance channels with afferent visual pathways may remain valid for simple detection tasks. The effects of epilepsy on these visual pathways needs to be investigated further with specific stimuli designed to independently stimulate them, although this was not the aim of the present research.

Decreases in visual CS in the EG was expected because this study measured visual CS in adults with generalized tonic-clonic seizure-type epilepsy, one of the most severe forms of epilepsy that changes several different areas of the nervous system (Kaplan \& Sandock, 1997; Lorenzato et al., 2002; Fonseca \& Tedrus, 2004). These data are consistent with previous reports that found epilepsy-related damage to the visual system and decreased CS (Nousiainen, Kalviainen, \& Mantyjarvi, 2000; Mirabela et al., 2007).

Our laboratory is developing new research to evaluate whether this damage is caused by the epilepsy itself or anticonvulsant treatment, for which no consensus has yet been reached (Tomson et al., 1988; Verrotti, Ciampani, Pomilio, Latini, \& Chiarelli, 2006). Mirabella et al. (2007) found reduced visual CS in children with infantile seizures who were not using any drugs. However, several other studies showed that anticonvulsant drugs, such as carbamazepine, alter visual function (Tomson et al., 1988; Sartucci et al., 1997; Steinhoff, Freudenthaler, \& Paulus, 1997). Nousiainen et al. (2000) did not find changes in visual function related to carbamazepine treatment in volunteers with epilepsy. The effects of anticonvulsants on visual perception and the nervous system need to be better analyzed to solve the contradictions found in the literature.

In the present study, the volunteers in the EG were all taking drugs, including carbamazepine, oxicarbazepine, and phenobarbital, for more than 4 years. Finding volunteers with generalized tonic-clonic seizure-type epilepsy who are not taking any medications is difficult. We found a significant decrease in visual CS related to epilepsy, but we cannot conclude that this was caused only by epilepsy or whether it was attributable to both epilepsy and anticonvulsant drugs.

In summary, evaluating the effects of epilepsy on visual CS using the psychophysical method is a valuable in vivo, noninvasive tool for assessing the effects of epilepsy and the drugs used to treat epilepsy patients on 
the central nervous system in humans. Such a method may also be useful for disease diagnosis, prognosis, and treatment follow-up.

\section{Acknowledgments}

The authors wish to thank all of the volunteers who participated in this research, the psychiatrists from the Centro de Atenção Psicossocial (CAPS) from João Pessoa, Paraíba, Brazil, who diagnosed the patients with epilepsy, and Conselho Nacional de Desenvolvimento Científico e Tecnológico (CNPq) and the Fundação de Amparo à Pesquisa do Estado de São Paulo (FAPESP) for financial support.

\section{References}

Burbeck, C.A., \& Kelly, D.H. (1981). Contrast gain measurements and the transient/sustained dichotomy. Journal of the Optical Society of America, 71, 1335-1342.

Campbell, F.W., \& Green, D.G. (1965). Optical and retinal factors affecting visual resolution. Journal of Physiology, 181, 576-593.

Fonseca, L.C., \& Tedrus, G.M.A.S. (2004). Conhecimentos e atitudes sobre epilepsia entre universitários da área da saúde. Arquivos de Neuropsiquiatria, 62, 1068-1073.

García-Pérez, M.A. (1998). Forced-choice staircases with fixed step sizes: asymptotic and small-sample properties. Vision Research, $38,1861-1881$.

Garzon, E. (2002). Epilepsia refratária: conceito e contribuição das novas drogas antiepilépticas e de outras modalidades terapêuticas. Revista Neurociências, 10(2), 66-82.

Guerreiro, C.A.M., \& Guerreiro M.M. (1996). Epilepsia (2nd edition). São Paulo: Lemos Editorial.

Hilton, E.J.R., Cubbidge, R.P., Hosking, S.L., Betts, T., \& Comaish, I.F. (2002). Patients treated with vigabatrin exhibit central visual function loss. Epilepsia, 43, 1351-1359.

Kaplan, I., \& Sandock, B.J. (1997). Compêndio de psiquiatria. Porto Alegre: Artes Médicas.

Lee, B.B. (2011). Visual pathways and psychophysical channels in the primate. Journal of Physiology, 589, 41-47.

Lorenzato, R.Z., Cavalli, R.C., \& Duarte, G. (2002). Epilepsia e gravidez: evolução e repercussões. Revista Brasileira de Ginecologia e Obstetrícia, 24, 521-526.

Michelson, A.A. (1891). On the application of interference methods to spectroscopic measurements: I. London, Edinburgh, and Dublin Philosophical Magazine and Journal of Science, 31, 338-346 and Plate VII.

Mirabela, G., Morong, S., Buncic, J.R., Snead, O.C., Logan, W.J., Weiss, S.K., \& Westall, C.A. (2007). Contrast sensitivity is reduced in children with infantile spasms. Investigative Ophthalmology and Visual Science, 48, 3610-3615.

Nousiainen, I., Kalviainen, R., \& Mantyjarvi, M. (2000). Contrast and glare sensitivity in epilepsy patients treated with vigabatrin or carbamazepine monotherapy compared with healthy volunteers. British Journal of Ophthalmology, 84, 622-625.

Pinel, J.P.J. (2005). Biopsicologia (5th edition). Porto Alegre: Artmed. Santo, J.L.E., Maineri, N., \& Portuguez, M.W. (2004). Epilepsia e crises não epilépticas. Journal of Epilepsy and Clinical Neurophysiology, 10, 29-33.

Santos, N.A., \& Alencar, C.C.G. (2010). Early malnutrition diffusely affects children contrast sensitivity to sinewave gratings of different spatial frequencies. Nutritional Neuroscience, 13, 189-194.

Santos, N.A., Alencar, C.C.G., \& Dias, Y.H.N. (2009). Contrast sensitivity function of sine-wave gratings in children with acute malnutrition. Psychology and Neuroscience, 2, 11-15.

Sartucci, F., Massetani, R., Galli, R., Bonanni, E., Tognoni, G., Milani, S., Iudice, A., \& Murri, L. (1997). Visual contrast sensitivity in carbamazepine-resistant epileptic patients receiving vigabatrin as add-on therapy. Journal of Epilepsy, 10(1), 7-11.

Schwartz, S.H. (2004). Visual perception: a clinical orientation (3rd edition). New York: McGraw Hill.

Slaghuis, W.L., \& Thompson, A.K. (2003). The effect of peripheral visual motion on focal contrast sensitivity in positive- and negativesymptom schizophrenia. Neuropsychologia, 41, 968-980.

Sorri, I., Kalviainen, R., \& Mantyjarvi, M. (2005a). Color vision and contrast sensitivity in epilepsy patients treated with initial tiagabine monotherapy. Epilepsy Research, 67, 101-107.

Sorri, I., Rissanen, E., Mantyjarvi M., \& Kalviainen, R. (2005b). Visual function in epilepsy patients treated with initial valproate monotherapy. Seizure, 14, 367-370.

Souza, G.S., Gomes, B.D., Saito, C.A., da Silva Filho, M., \& Silveira, L.C.L. (2007). Spatial luminance contrast sensitivity measured with transient VEP: comparison with psychophysics and evidence of multiple mechanisms. Investigative Ophthalmology and Visual Science, 48, 3396-3404.

Steinhoff, B.J., Freudenthaler, N., \& Paulus, W. (1997). The influence of established and new antiepileptic drugs on visual perception: II. A controlled study in patients with epilepsy under long-term antiepileptic medication. Epilepsy Research, 29, 49-58.

Tedrus, G.M.A.S., \& Fonseca, L.C. (2005). Epilepsias idiopáticas focais occipitais da infância: estudo de 63 casos. Arquivos de Neuropsiquiatria, 63, 61-67.

Tomson, T., Nilsson, B.Y., \& Levi, R. (1988). Impaired visual contrast sensitivity in epileptic patients treated with carbamazepine. Archives of Neurology, 45, 897-900.

Verrotti, A., Ciampani, M., Pomilio, M., Latini, G., \& Chiarelli, F. (2006). Endocrine abnormalities induced by antiepileptic drugs. Current Pediatric Reviews, 2(1), 85-92.

Verrotti, A., Manco, R., Matricardi, S, Franzoni, E., \& Chiarelli, F. (2007). Antiepileptic drugs and visual function. Pediatric Neurology, 36, 353-360.

Wesner, M.F., \& Tan, J. (2006). Contrast sensitivity in seasonal and nonseasonal depression. Journal of Affective Disorders, 95, 19-28.

Wetherill, G.B., \& Levitt, H. (1965). Sequential estimation of points on a psychometric function. British Journal of Mathematical and Statistical Psychology, 18, 1-10.

World Health Organization - WHO. Relatório Sobre a Saúde no Mundo (2010) - Organização Panamericana da Saúde -Organização Mundial de Saúde - Genève - Swiss.

Yacubian, E.M.T. (1999). Tratamento medicamentoso das epilepsias. São Paulo: Lemos Editorial. 